\title{
Molar pregnancy with a coexisting live fetus as a challenge for perinatology. A mini-review with two case reports
}

\author{
Michał Ciebiera, Cezary Wojtyła, Grzegorz Jakiel, Tomasz Roszkowski \\ First Department of Obstetrics and Gynecology, Centre of Postgraduate Medical Education, Warsaw, Poland
}

\begin{abstract}
Coexistence of a molar pregnancy with a live fetus is a rare and complex clinical condition. Twin pregnancies with a healthy fetus and a hydatidiform mole arise in one in 20,000-100,000 pregnancies, depending on data. This article reviews available literature on pregnancies with coexistence of a hydatidiform mole and a live fetus, and presents two case reports. Successful diagnosis is based on multiple elements e.g. experience in sonography and prenatal diagnosis. There are no clear guidelines for how to proceed with this pathology, therefore the management strategies remain uncertain. Parents must be aware of increased risk of intrauterine fetal growth restriction, fetal distress, premature labor, preeclampsia, thyrotoxicosis, hemorrhage, and gestational trophoblastic neoplasia associated with this condition. Management in these pregnancies depends on the individual expectation of the pregnant woman, and parents should be made aware of the low probability of favourable outcome for the fetus. A large case series of molar pregnancies with a coexisting live fetus could be useful to establish evidence-based management guidelines.
\end{abstract}

KEY WORDS: twin pregnancy, hydatidiform mole, molar pregnancy, molar pregnancy with coexisting fetus.

ADDRESS FOR CORRESPONDENCE: Michał Ciebiera, MD, PhD, I Department of Obstetrics and Gynecology, Centre of Postgraduate Medical Education, 231 Czerniakowska Street, 00-416 Warsaw, Poland, phone: +48 607155 177, e-mail: michal.ciebiera@gmail.com

\section{INTRODUCTION}

Partial or complete hydatidiform moles arise in approximately one in 1,000 pregnancies [1]. The first of these, a partial hydatidiform mole (PHM), is composed of chorionic and embryonic tissue. It occurs due to fertilization of an oocyte by two sperm resulting in a triploid karyotype (69 chromosomes). The second, complete hydatidiform mole (CHM), occurs when the empty oocyte is fertilized by one or two sperm. In general, CHM has a diploid karyotype (46 chromosomes; $46, \mathrm{XY}$ or $46, \mathrm{XX})$, but a $23, \mathrm{X}$ karyotype is rare [2]. If each pair of chromosomes is identical, this mole is homozygous (with karyotype 46, XX). The second mechanism, in which the empty oocyte fuses with two different spermatic genomes (dispermy), will result in a heterozygous mole (karyotype can be $46, \mathrm{XX}$ or $46, \mathrm{XY}$ ) [2]. It should be noted that partial and complete molar pregnancies are separate pathologic entities $[3,4]$.

The coexistence of a molar pregnancy with a twin live fetus is a rare and complex clinical condition. Twin pregnancies with a healthy fetus and hydatidiform mole arise in one in 20,000-100,000 pregnancies, depending on data $[1,5,6]$. It has been suggested that there is an increased incidence of twin pregnancies with a coexisting mole and fetus in patients who have undergone pharmacological induction of ovulation before pregnancy [7].

There are three types of pregnancies that result in coexistence of a live fetus with molar tissue $[5,6]$ : 1) CHMCF, dichorionic twin pregnancy with a complete hydatidiform mole (46 chromosomes, all paternal) and a normal fetus (46 chromosomes, 23 maternal and 23 paternal); 2) PHMCF, singleton pregnancy consist- 
ing of a triploid fetus with a partial hydatidiform mole placenta, and 3) coexistence of a blighted ovum with hydropic changes and a normal fetus. PHMCF is reported rarely. It is thought that the etiological risk factors for partial mole are prior abortion(s), an irregular cycle, and a maternal age over 40 years $[1,8]$.

Hydatidiform mole with a live fetus is associated with a higher risk of severe pregnancy complications, in particular vaginal bleeding, preeclampsia (PE), hyperthyroidism, intrauterine demise and gestational trophoblastic neoplasia (GTN) $[5,8,9]$. Management and diagnosis of these patients is challenging. The fetus can be normal in a twin pregnancy like CHMCF, but continuation of pregnancy is often associated with maternal complications leading to clinical complications for the pregnant woman or the fetus $[5,6]$.

\section{MATERIAL AND METHODS}

This article is a review of available literature about pregnancies with coexistence of hydatidiform moles with a live fetus, as well as the management strategies. Two case studies from I Department of Obstetrics and Gynecology, Centre of Postgraduate Medical Education are presented.

\section{CASE STUDY 1}

The patient was a 40-year-old woman (gravida 2, parity 2) with previous vaginal delivery of a healthy infant. In early pregnancy, the patient was diagnosed with a singleton pregnancy. During the $8^{\text {th }}$ week of gestation the patient reported heavy vaginal bleeding which was treated with the use of dydrogesterone. Ultrasound examination at 12 weeks of gestation (PAPP-A and free beta-hCG serum examination) revealed a high risk $(1: 7)$ of trisomy 21, a free beta-hCG level of $296.7 \mathrm{mIU} / \mathrm{ml}$ (9.179 MoM), and a normal placenta. Amniocentesis in the $16^{\text {th }}$ week of gestation revealed a normal male karyotype $(46, \mathrm{XY})$ by multiplex ligation-dependent probe amplification (MLPA) as well as in standard culture. Part of the placenta was described as abnormal in the ultrasound scan, with the suspicion of molar pregnancy (Fig. 1). The high risk of a negative outcome was carefully discussed with the patient, who decided to continue the pregnancy. After the $20^{\text {th }}$ week of gestation, the patient had a clinical consultation as well as an ultrasound scan every 2 weeks. At every visit, the patient had cyclic checks of thyroid hormone and beta-hCG levels, as well as blood and urine examinations. The patient was advised to measure her blood pressure four times a day. During the $34^{\text {th }}$ week of pregnancy the patient was hospitalized due to oligohydramnios (maximum vertical pocket under $2 \mathrm{~cm}$ ). A standard full course of steroids (betamethasone) was administered. The patient had an elective cesarean section at 35 weeks gestation. A live male late preterm infant (2160 g) with an Apgar
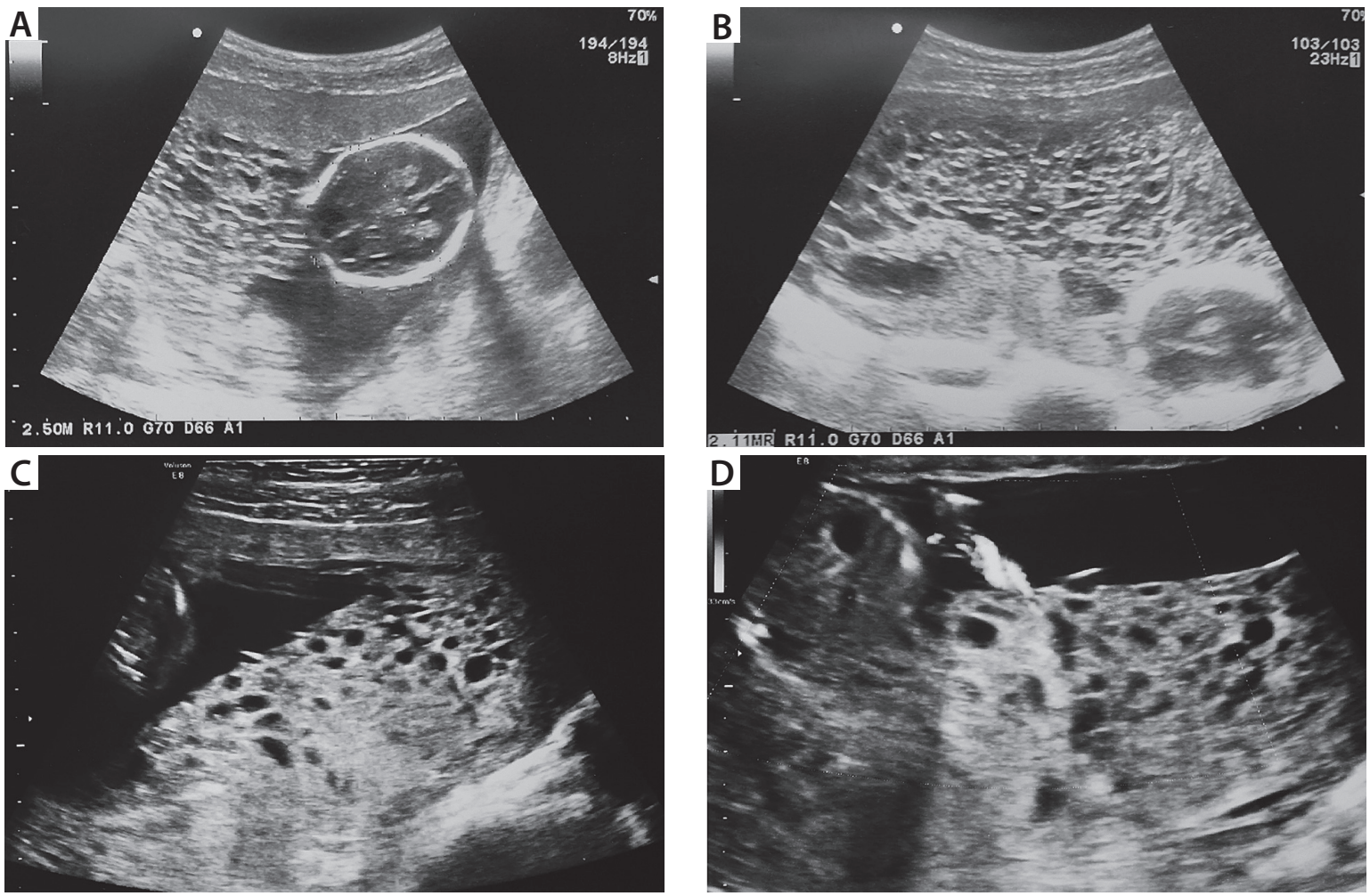

FIG. 1. Case study 1 and 2 - ultrasound scans. A) Case study 1. 16 $6^{\text {th }}$ week of gestation, molar tissue, healthy fetus head. B) Case study 1. $16^{\text {th }}$ week of gestation, molar tissue. C) Case study 2. $14^{\text {th }}$ week of gestation. Healthy fetus and molar tissue. D) Case study 2. 14 $4^{\text {th }}$ week of gestation. Molar tissue 

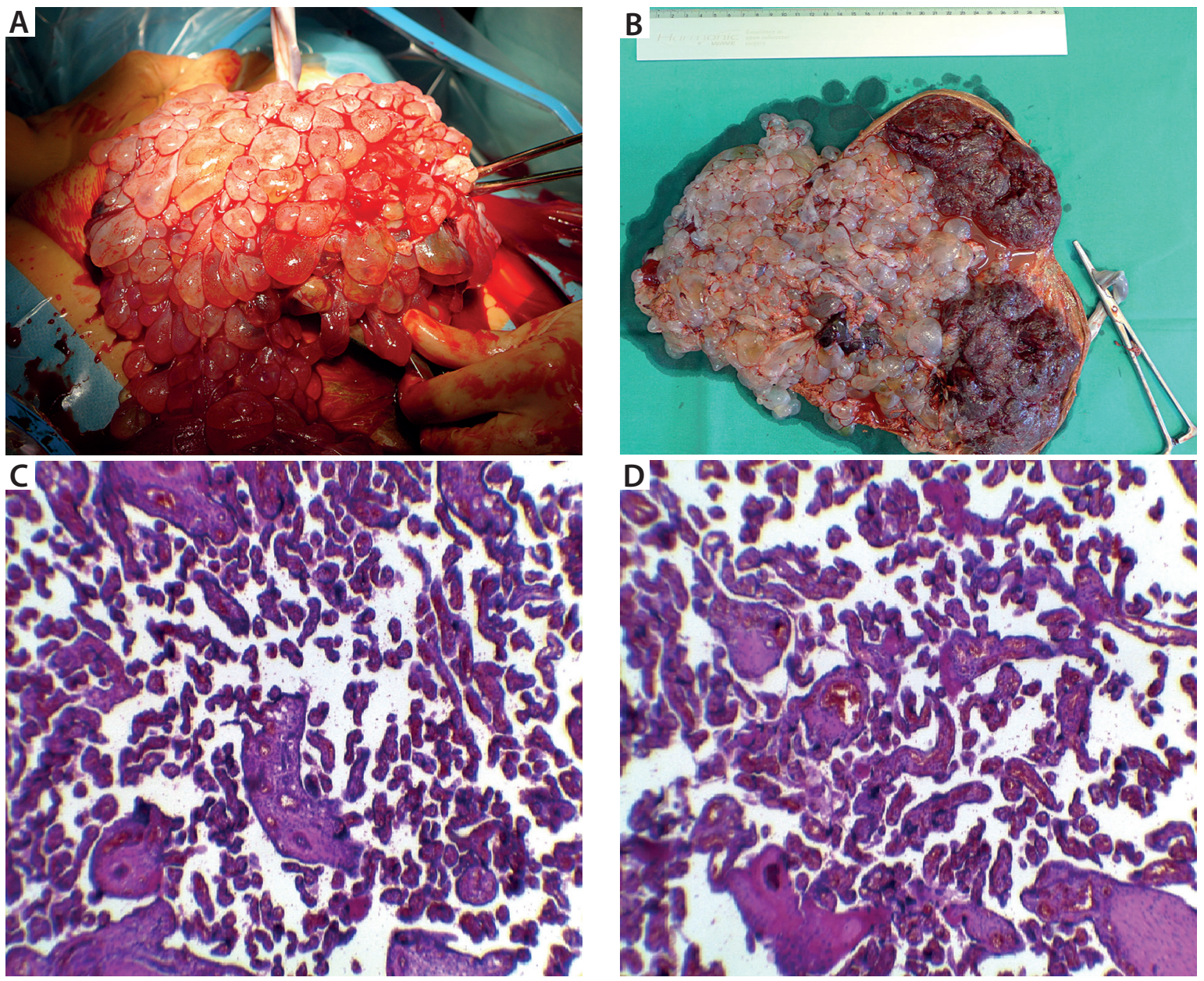

FIG. 2. Case study 1. A, B) Molar and normal placenta. C, D) Microscopic findings. H\&E staining. Magnification 4X

score of 9 at $1 \mathrm{~min}$ and 10 at $5 \mathrm{~min}$ was delivered. Both normal and molar placentas were extracted completely. The microscopic findings confirmed a complete mole (Fig. 2). After the cesarean section, the patient had a serum beta-hCG level of 200,998 $\mathrm{mIU} / \mathrm{ml}$. The next day the level had dropped to $44,827 \mathrm{mIU} / \mathrm{ml}$. At the 6-month follow-up there were no signs of GTN.

\section{CASE STUDY 2}

The second patient was a 25 -year-old woman (gravida 1 , parity 1$)$. In the $8^{\text {th }}$ week of gestation the patient was diagnosed with a singleton pregnancy. An ultrasound examination at 12 weeks revealed abnormal multicystic findings in the placenta. Amniocentesis was performed at 16 weeks gestation, and a normal female karyotype (46, XX) was revealed by both MLPA and standard culture. Because of the abnormal placental findings, the suspicion of molar pregnancy was raised (Fig. 1). The risk of negative outcomes and complications was carefully discussed with the patient, who decided to continue the pregnancy. All procedures were performed as in the previously described case. The patient was diagnosed with gestational diabetes mellitus after an oral glucose tolerance test. A diabetic diet was implemented with a good effect. In the $31^{\text {st }}$ week of gestation, preterm prelabor rupture of the membranes occurred. A full course of steroids was administered. Three days later, the patient underwent an emergency cesarean section because of uterine contractions and nonreactive CTG. A live female infant $(1500 \mathrm{~g})$ with an Apgar score of 6 at $1 \mathrm{~min}$ and 8 at 5 min was delivered. The placentas were extracted completely. The microscopic findings confirmed a complete mole (Fig. 3). After the cesarean section, the patient had a serum beta-hCG level of $131543 \mathrm{mIU} / \mathrm{ml}$, which had dropped to $24887 \mathrm{mIU} / \mathrm{ml}$ the following day. The betahCG level after 3 months had normalized completely. No signs of GTN were noted during follow-up.

\section{DISCUSSION \\ DIAGNOSIS}

Successful diagnosis is based on multiple elements including clinical symptoms, biology, ultrasonography, and genetics. Sometimes differentiation can only be made during the postpartum or termination period $[10,11]$. A hydatidiform mole and coexisting fetus can be diagnosed during the first trimester, but this is difficult. A molar placenta is often described as a "snowstorm", as it is characterized by multiple oval cysts (grape bunches) 
sized from 1 to $30 \mathrm{~mm}$. In CHMCF, these lesions are described together with a coexisting fetus and normal placenta $[12,13]$. Unfortunately, not all sonographers pay sufficient attention to the placental echogenicity, especially in the first trimester scan. This is why ultrasonography can miss molar changes in about $40 \%$ of cases [14]. On the other hand, according to available data, only $30 \%$ of partial moles are reported to be detected by ultrasound $[15,16]$.

Partial moles have a heterogenous form with multicystic spaces mixed with areas of solid tissue. Doppler examination shows a high-velocity and low impedance of flow in the vessels surrounding these irregular masses [12]. Differential diagnosis may be difficult as partial moles do not have typical characteristics like those of a complete hydatidiform mole, such as a "snowstorm" appearance, which is often absent in a partial molar pregnancy. Differentiation of partial molar pregnancy from CHMCF is absolutely crucial. In most partial molar pregnancies, the fetus has a triploid karyotype and presents anatomical malformations. When the dip- loid karyotype is present, we can expect a normal fetus with a normal placenta, as well as the molar pregnancy (excluding any associated anatomical malformations and genetic findings).

Molar pregnancies coexisting with live fetuses require a high grade of suspicion and fast differential diagnosis. In our opinion, in the case of incorrect placenta imaging, combined screening is a very useful tool. Abnormally high beta-hCG levels should be evaluated and invasive procedures such as amniocentesis should be implemented. Rapid genetic analyses can be additionally advised. It should be noted, however, that some of these are not able to diagnose triploidy (e.g., Multiplex Ligation-dependent Probe Amplification [MLPA]).

Invasive diagnostics with the use of chorionic villus sampling (CVS), amniocentesis, or fetal cord blood sampling enables clinicians to distinguish between diploid and triploid fetuses. A partial mole can be detected by DNA polymorphism analysis. In addition, CVS can also be performed on molar tissue, in which an absence of maternal alleles will confirm a diandrogen-
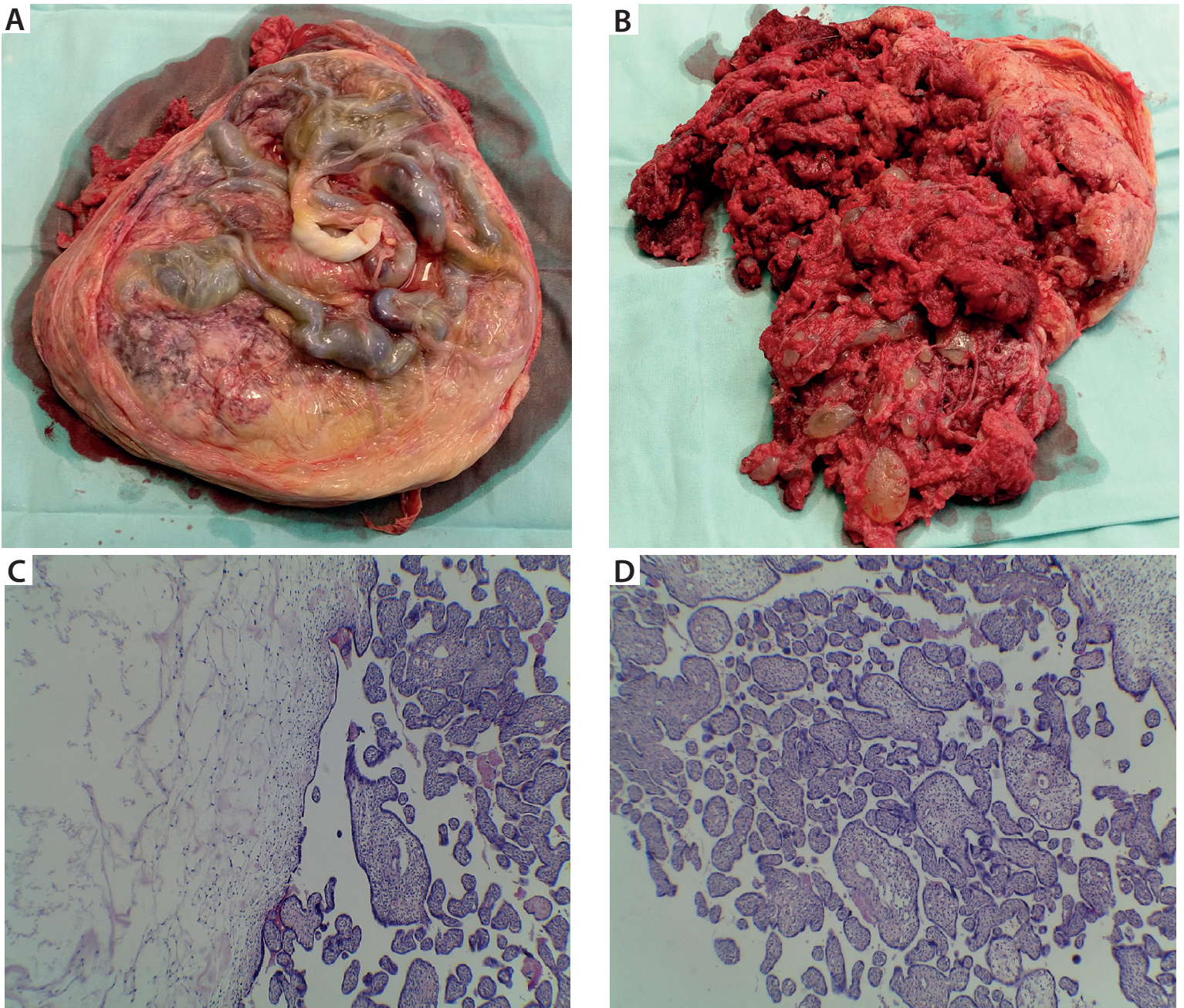

FIG. 3. Case study 2. A, B) Molar and normal placenta. C, D) Microscopic findings. H\&E staining. Magnification 40X 
ic complete mole [17]. Karyotyping is essential when deciding whether to continue the pregnancy presuming the outcome. A triploid karyotype indicates a triploid fetus, and termination of pregnancy (TOP) should be recommended in these cases. A diploid fetal karyotype generally indicates a viable fetus with a coexisting molar pregnancy as a twin. In these cases, the pregnancy can be allowed to continue as there is considerable chance that a normal neonate will result [18]. It is important to remember that a diploid karyotype is not a confirmed diagnosis of CHMCF. In these cases, DNA polymorphism analysis is the most reliable method. While CHMCF is considered as dizygotic, cases of mosaicism resulting in $\mathrm{CHM}$ and a normal fetus have been described. Microsatellite marker analyses have proven that CHMCF can be monozygotic in one out of seven cases [19]. Some studies suggest that the immunohistochemical staining of p57 can distinguish androgenetic moles from biparental ones (normal fetus, partial mole) as p57 is an imprinted gene with preferential expression on the maternal allele $[20,21]$. The data regarding cellfree DNA and hydatidiform moles are still limited, but in our opinion this could be a breakthrough in the diagnosis of these pregnancies [22].

There are several differential diagnoses other than partial mole, and these include mesenchymal dysplasia, choriocarcinoma, placenta circumvallate, and placental hematoma or multiple hematomas. Confirmation of diagnosis is based on histopathological examination. The differentiation between choriocarcinoma and molar pregnancy can be difficult. However, despite this being a rare pathology, it should always be investigated and excluded. Choriocarcinoma is usually diagnosed based on the high level of vascularization [12]. Placental hematoma may mimic partial mole with its mixture of solid tissue and cystic sonographic appearance. Furthermore, differentiation in cases of mesenchymal dysplasia can be very difficult.

\section{MANAGEMENT}

The management of $\mathrm{CHMCF}$ remains ambiguous as there are no clear guidelines on how to proceed with these cases. The greatest challenge for the clinician is the accurate evaluation of maternal bleeding and hypertension risk, as well as the likelihood that the infant will be born alive and in good condition $[9,23]$.

Many molar pregnancies are referred to tertiary centers after the combined first trimester screening shows high levels of free beta-hCG. It is important to remember that serum beta-HCG levels should be highest at the beginning of the second trimester. An abnormally fast elevation in beta-hCG level in the second trimester is a major risk factor for future TOP [24]. According to Sebire et al., TOP should be considered when the serum beta-hCG level remains greater than $106 \mathrm{mIU} / \mathrm{ml}$ [1]. Usually pregnancies that end with a live birth show a decline in serum beta-hCG levels after the beginning of the second trimester. In these pregnancies, the size of the molar part of the placenta declines until labor [24]. Wee et al. advises bimonthly monitoring of the betahCG level as well as an ultrasound scan in these cases [23]. A progressive decrease in serum beta-HCG level and a normal fetal karyotype are necessary for management to an age of fetal viability [25].

All women with a CHMCF pregnancy should be counseled about the risk of PE, thromboembolic disease, hemorrhage, hyperemesis gravidarum and hyperthyroidism, as well as fetal complications including miscarriage, stillbirth and preterm labor. Gestational trophoblastic neoplasia must be considered as it represents a severe complication of molar pregnancies [8]. Serial thyroid gland tests and blood and urine examinations are a must when managing these pregnancies, as they can show if the patient is developing thyrotoxicosis or proteinuria among other conditions, or whether they require additional therapy.

\section{COMPLICATIONS}

Even if the fetus and placenta are thought to be normal, continuation of the pregnancy is decided depending on the associated complications [26]. Parents must be aware of fetal growth retardation, fetal distress, premature delivery, $\mathrm{PE}$, thyrotoxicosis, hemorrhage, intrauterine fetal demise, GTN and fetal malformations. It is never certain whether the pregnancy can be continued safely $[6,13,15,27]$.

The most frequently reported complications of CHMCF are vaginal bleeding, PE, preterm labor, and GTN. In CHMCF pregnancies, an estimated 30-40\% result in live term births. The risk of severe PE is $6 \%$, which is no higher than in singleton CHM, but slightly higher than for normal twin pregnancies. The rate of GTN is estimated at between $30 \%$ and $50 \%[6,11,13$, 27, 28]. According to Niemann et al., the risk of GTN is the same for complete singleton molar pregnancy and CHMCF [29]. Data reported by Sebire et al. showed that the risk of GTN did not differ between women undergoing first trimester TOP and those who continued their pregnancies [1]. Stillbirth appears to be very common in CHMCF, mostly before 24 weeks of gestation. After that time, the risk of stillbirth depends on the serum beta-HCG level as well as whether any additional complications occur [24]. When severe complications occur, management generally involves TOP in order to avoid potential complications. As $\mathrm{PE}$ is especially indicative of a poor outcome, early onset of PE must be considered an indication for TOP [15]. Low-dose acetylsalicylic acid has not yet been examined for the prevention of PE in molar pregnancies, although it seems that it could be helpful in some cases.

In 2002, Sebire et al. described 77 cases of CHMCF. Of these, 24 women decided to have an abortion and 
53 women chose to continue their pregnancies. Twenty-three of those pregnancies resulted in abortion or intrauterine death (before the $24^{\text {th }}$ week), and eight fetal deaths occurred after that period. Overall, 20 of the 77 women (38\%) delivered a baby, with a median gestational age of 35 weeks. Most of those pregnancies were delivered beyond 32 weeks, avoiding the risks of extreme prematurity. This study also showed that about $60 \%$ of continued CHMCF pregnancies resulted in either intrauterine death of the co-twin or spontaneous pregnancy loss [1].

In the same study, 15 of the 77 women developed GTN (19\%) [1]. There was no significant difference in the risk of developing GTN between patients who had a TOP in the first trimester and those who terminated the pregnancy in the second trimester. Niemann et al. reported a similar risk of about 25\% [29] to 50\% [28], and even up to $57 \%$ [11]. Ovulation stimulation was identified as a key feature in the patients' history which significantly increased the risk of GTN. In a study by Massardier et al., 4 of 14 (28\%) patients had undergone ovulation stimulation, and all those cases progressed to a GTN [28]. Wee et al. also reported the same rate [23]. Chest X-ray and abdominal ultrasound scan are necessary when diagnosing GTN. Furthermore, weekly monitoring of serum beta-hCG during the postpartum period provides the main indicator of trophoblast activity [16]. Chemotherapy is indicated for the management of GTN, especially in cases with a persistent high beta-hCG level 6 months after evacuation or in those with histopathological diagnosis of choriocarcinoma $[8,30]$. If the patient decides to continue the pregnancy, serial serum beta-hCG level measurements and a chest X-ray are critical to screen for GTN and metastases. Post-evacuation follow-up is necessary. Quantitative serum beta-hCG assay is recommended within 48 hours of evacuation and every 1 to 2 weeks while elevated, then monthly for minimum of 6 months after the beta-hCG levels have returned to normal.

\section{CONCLUSIONS}

The possible maternal and fetal complications derived to molar pregnancy must be discussed with the patient before they decide whether to continue the pregnancy. Early TOP has traditionally been advised, although not all women will consider this option. The outcome of the pregnancy depends on the molar component. In the first option, the molar part of the pregnancy becomes quiescent allowing the pregnancy to continue. In the second option, the molar part continues to grow, leading to severe maternal or fetal complications. In cases where a normal fetal karyotype is diagnosed, the elevation in beta-hCG level slows down and serious pathology is absent, waiting until fetal viability is achieved can be proposed. Management of CHMCF depends mostly on each pregnant woman's individual expectation.
The women must be aware of the risks and complications and of the low favorable outcome for the fetus (between $30 \%$ to $50 \%$ depending on data). Pregnant women should be informed of the potentially high risk of developing GTN in pregnancy, even if they decides to terminate the pregnancy. An individualistic, well-discussed approach is probably the best option, depending upon factors predicting the likely outcome. The main factors to be considered in this decision-making process include the stage of pregnancy at which the patient is diagnosed, the availability of prenatal diagnostic methods (ultrasound, invasive testing, DNA polymorphism), the viability of the fetus, whether the patient can access help if preterm labor occurs, whether the patient can be closely supervised at a tertiary center, and the absence of other risk factors. No favorable outcomes of PHMCF have been described, therefore TOP should be advised in all cases of PHMCF. Regarding CHMCF, a genetic test should be performed to confirm a normal karyotype, and if there are no other abnormalities detected in the ultrasound, pregnancy may be continued as long as maternal complications are absent. In case of complications like hypertension or hyperthyroidism, treatment should be attempted. The decision regarding continuation should be based on the availability of effective treatment. During the postpartum period, intensive follow-up should be implemented with serial beta-hCG level measurements and radiologic examinations.

Women who decide to continue the pregnancy must be made aware of the significantly high risk of maternal morbidity and hysterectomy as the pregnancy progresses. However, there is still a lack of data on this topic, therefore a large case series of CHMCF patients could be useful to establish reliable evidence-based management guidelines.

\section{ACKNOWLEDGEMENTS}

This study was funded by Centre of Postgraduate Medical Education. Grant number 501-1-21-27-17.

Special thanks to all clinicians who were involved in the diagnostic and therapeutic process of described patients.

\section{DISCLOSURE}

The authors report no conflict of interest.

\section{References}

1. Sebire NJ, Foskett M, Paradinas FJ, et al. Outcome of twin pregnancies with complete hydatidiform mole and healthy co-twin. Lancet 2002; 359: 2165-2166.

2. Kaiji T, Ohama K. Androgenetic origin of hydatidiform mole. Nature 1977; 268: 633-634.

3. Szulman AE, Surti U. The syndromes of hydatidiform mole. I. Cytogenetic and morphologic correlations. Am J Obstet Gynecol 1978; 131: 665-671. 
4. Szulman AE, Surti U. The syndromes of hydatidiform mole. II. Morphologic evolution of the complete and partial mole. Am J Obstet Gynecol 1978; 132: 20-27.

5. Vaisbuch E, Ben-Arie A, Dgani R, et al. Twin pregnancy consisting of a complete hydatidiform mole and co-existent fetus: report of two cases and review of literature. Gynecol Oncol 2005; 98: 19-23.

6. Dolapcioglu K, Gungoren A, Hakverdi S, et al. Twin pregnancy with a complete hydatidiform mole and co-existent live fetus: Two case reports and review of the literature. Arch Gynecol Obstet 2009; 279: 431-436.

7. Shazly SA, Ali MK, Abdel Badee AY, et al. Twin pregnancy with complete hydatidiform mole and coexisting fetus following ovulation induction with a non-prescribed clomiphene citrate regimen: a case report. J Med Case Rep 2012; 6: 95.

8. Seckl MJ, Sebire NJ, Berkowitz RS. Gestational trophoblastic disease. Lancet 2010; 376: 717-729.

9. Lurain JR. Gestational trophoblastic disease I: epidemiology, pathology, clinical presentation and diagnosis of gestational trophoblastic disease, and management of hydatidiform mole. Am J Obstet Gynecol 2010; 203: 531-539.

10. Cohen MC, Roper EC, Sebire NJ, et al. Placental mesenchymal dysplasia associated with fetal aneuploidy. Prenat Diagn 2005; 25: 187-192.

11. Fishman DA, Padilla LA, Keh P, et al. Management of twin pregnancies consisting of a complete hydatidiform mole and normal fetus. Obstet Gynecol 1998; 91: 546-550.

12. Vaisbuch E, Romero R, Kusanovic JP, et al. Three-dimensional sonography of placental mesenchymal dysplasia and its differential diagnosis. J Ultrasound Med 2009; 28: 359-368.

13. Vimercati A, de Gennaro AC, Cobuzzi I, et al. Two cases of complete hydatidiform mole and coexistent live fetus. J Prenat Med 2013; 7: 1-4

14. Lindholm H, Flam F. The diagnosis of molar pregnancy by sonography and gross morphology. Acta Obstet Gynecol Scand 1999; 78: 6-9.

15. Matsui H, Sekiya S, Hando T, et al. Hydatidiform mole coexistent with a twin live fetus: A national collaborative study in japan. Hum Reprod 2000; 15: 608-611.

16. Marcorelles P, Audrezet MP, Le Bris MJ, et al. Diagnosis and outcome of complete hydatidiform mole coexisting with a live twin fetus. Eur J Obstet Gynecol Reprod Biol 2005; 118: 21-27.

17. Wax JR, Pinette MG, Chard R, et al. Prenatal diagnosis by DNA polymorphism analysis of complete mole with coexisting twin. Am J Obstet Gynecol 2003; 188: 1105-1106.

18. Montes-de-Oca-Valero F, Macara L, Shaker A. Twin pregnancy with a complete hydatidiform mole and co-existing fetus following in-vitro fertilization: case report. Hum Reprod 1999; 14: 2905-2907.

19. Niemann I, Bolund L, Sunde L. Twin pregnancies with diploid hydatidiform mole and co-existing normal fetus may originate from one oocyte. Hum Reprod 2008; 23: 2031-2035.

20. Crisp H, Burton JL, Stewart R, Wells M. Refining the diagnosis of hydatidiform mole: Image ploidy analysis and p57kip2 immunohistochemistry. Histopathology 2003; 43: 363-373.
21. Kihara M, Matsui H, Seki K, et al. Genetic origin and imprinting in hydatidiform moles. Comparison between DNA polymorphism analysis and immunoreactivity of p57kip2. J Reprod Med 2005; 50: 307-312.

22. Openshaw MR, Harvey RA, Sebire NJ, et al. Circulating cell free DNA in the diagnosis of trophoblastic tumors. EBioMedicine 2016; 4: 146-152.

23. Wee L, Jauniaux E. Prenatal diagnosis and management of twin pregnancies complicated by a co-existing molar pregnancy. Prenat Diagn 2005; 25: 772-776.

24. Bristow RE, Shumway JB, Khouzami AN, Witter FR. Complete hydatidiform mole and surviving coexistent twin. Obstet Gynecol Surv 1996; 51: 705-709.

25. Lee SW, Kim MY, Chung JH, et al. Clinical findings of multiple pregnancy with a complete hydatidiform mole and coexisting fetus. J Ultrasound Med 2010; 29: 271-280.

26. Sanchez-Ferrer ML, Machado-Linde F, Martinez-Espejo Cerezo A, et al. Management of a dichorionic twin pregnancy with a normal fetus and an androgenetic diploid complete hydatidiform mole. Fetal Diagn Ther 2013; 33: 194-200.

27. Albayrak M, Ozer A, Demir OF, et al. Complete mole coexistent with a twin fetus. Arch Gynecol Obstet 2010; 281: 119-122.

28. Massardier J, Golfier F, Journet D, et al. Twin pregnancy with complete hydatidiform mole and coexistent fetus: obstetrical and oncological outcomes in a series of 14 cases. Eur J Obstet Gynecol Reprod Biol 2009; 143: 84-87.

29. Niemann I, Sunde L, Petersen LK. Evaluation of the risk of persistent trophoblastic disease after twin pregnancy with diploid hydatidiform mole and coexisting normal fetus. Am J Obstet Gynecol 2007; 197: e41-45.

30. Committee on Practice Bulletins-Gynecology. ACOG practice bulletin \#53. Diagnosis and treatment of gestational trophoblastic disease. Obstet Gynecol 2004; 103: 1365-1377.

\section{AUTHORS' CONTRIBUTIONS}

MC, GJ prepared the research concept and collected data of the publication. All authors analysed data, wrote the article and finally approved it. GJ critically reviewed the article. 